\title{
Tunable Silicon Etalon for Simultaneous Spectral Filtering and Wavelength Monitoring of a DWDM Transmitter
}

\author{
Tapio Niemi, Maria Uusimaa, Simo Tammela, Päivi Heimala, Timo Kajava, Matti Kaivola, and Hanne Ludvigsen
}

\begin{abstract}
A temperature-tunable Fabry-Perot filter made of silicon has been developed and applied for simultaneous spectral filtering and wavelength monitoring of a directly modulated DFB laser. This novel device decreases the dispersion penalty of a costeffective dense wavelength-division-multiplexing (DWDM) transmitter in a long-haul transmission link. Moreover, it allows the operating wavelength of the transmitter to be accurately monitored in real time.
\end{abstract}

Index Terms-Optical communications, optical filters, optical transmitter.

\section{INTRODUCTION}

$\mathbf{T}$ O TRANSMIT optical signals in long-haul dense wavelength-division-multiplexing (DWDM) networks, directly modulated DFB lasers are often used due to their low-cost and straightforward implementation. The performance of these lasers is, however, often limited by frequency chirping, which results in pulse broadening in a dispersive single-mode fiber. Another limiting effect is imposed by wavelength drifts due to aging of the laser, which calls for a method to continuously track the wavelength of the transmitter.

Recently, temporal reshaping of the optical pulses by filtering the output of a directly modulated transmitter has been demonstrated to significantly decrease the dispersion penalty. In these experiments, either a Fabry-Perot interferometer or a fiber Bragg grating (FBG) were adopted [1]-[4]. The improvement of the quality of the transmitted signals by spectral filtering is essentially due to two effects. The extinction ratio of a signal having adiabatic frequency chirp becomes improved by transmitting the signal through a spectral filter with positively sloping transmission spectrum. The relaxation oscillations at the edges of the pulse are efficiently filtered out when the operation point is chosen close to the maximum of the filter passband.

In this letter, we report on the development of a tunable optical filter for simultaneous spectral filtering and wavelength monitoring of the output of a directly modulated DFB laser. The

\footnotetext{
Manuscript received July 5, 2000; revised September 26, 2000. This work was supported by The National Technology Agency (TEKES) through the ETX technology program.

T. Niemi, M. Uusimaa, and H. Ludvigsen are with Metrology Research Institute, Helsinki University of Technology, FIN-02015 HUT, Finland.

S. Tammela and P. Heimala are with VTT Electronics, Microelectronics, FIN02044 VTT, Finland.

T. Kajava and M. Kaivola are with Department of Engineering, Physics, and Mathematics, Helsinki University of Technology, FIN-02015 HUT, Finland.

Publisher Item Identifier S 1041-1135(01)00493-1.
}

filter is made of silicon [5], which is transparent in the wavelength range of 1.2-1.7 $\mu \mathrm{m}$. Moreover, the refractive index of silicon is sensitive to temperature. This allows the transmission of the filter to be tuned and locked to a preselected value on the slope of the filter transmission spectrum by controlling the optical thickness of the etalon via temperature. In our filter, this is realized with a heating resistor deposited on the silicon surface. By tracking the temperature of the etalon locked to a constant transmission value, changes in the wavelength of the transmitter may conveniently be monitored.

The silicon filter offers a flexible way to improve the long-haul performance of a directly modulated laser transmitter through temporal reshaping of the signal. Moreover, its operation principle allows a straightforward means for real-time wavelength tracking. Contrary to commercially available fixed-frequency filters, the operation of the device is not restricted to the ITU-channel grid, but the filter can be dynamically tuned over a wide wavelength range. In addition, the construction of the filter out of silicon wafer allows a cost-effective and reliable device implementation.

\section{Properties OF the TUNABle Filter}

The Fabry-Perot filter was fabricated by depositing dielectric mirrors on a double-side polished silicon wafer. The high-reflectivity mirrors were produced by depositing three $\lambda / 4$-layer pairs of $\mathrm{Si}_{3} \mathrm{~N}_{4}$ and $\mathrm{SiO}_{2}$ on both sides of the $380-\mu \mathrm{m}$-thick wafer [6]. The reflectivity of the mirrors $(R=0.66)$ and the free spectral range of the filter $(F S R=110 \mathrm{GHz})$ were determined by fitting an Airy-function to the measured transmission data (see Fig. 1). The $-3-\mathrm{dB}$ bandwidth of the filter is $\sim 14 \mathrm{GHz}$, which has been found to be suitable for this application [1].

The temperature tuning of the filter is realized by feeding current through a thin-film resistor deposited on the surface of the chip. The ring-shaped resistor made of molybdenum has a nominal resistance of $\sim 35 \Omega$. Tuning of the filter by temperature is feasible due to the strong temperature dependence of the refractive index of silicon. The temperature coefficient of the refractive index is $\sim 1.5 \cdot 10^{-4} 1 / \mathrm{K}$, which is almost two orders of magnitude larger than the temperature expansion coefficient of silicon. The filter chip with a size of $10 \mathrm{~mm} \times 5 \mathrm{~mm}$ was inserted in the air gap of a fiber-optic beam expander. A small portion of the optical power was tapped off before and after the filter with fiber couplers. The tapped-off powers were used to generate a feedback current for maintaining the ratio of the measured optical powers at a selected constant value. The total loss due to 


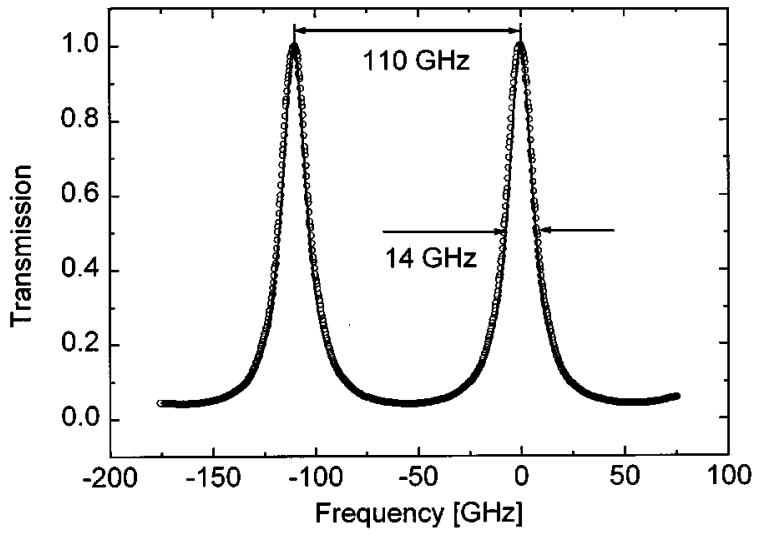

Fig. 1. Transmission spectrum of the Fabry-Perot filter. Open circles represent the measured data, and solid line is the fitted Airy function.

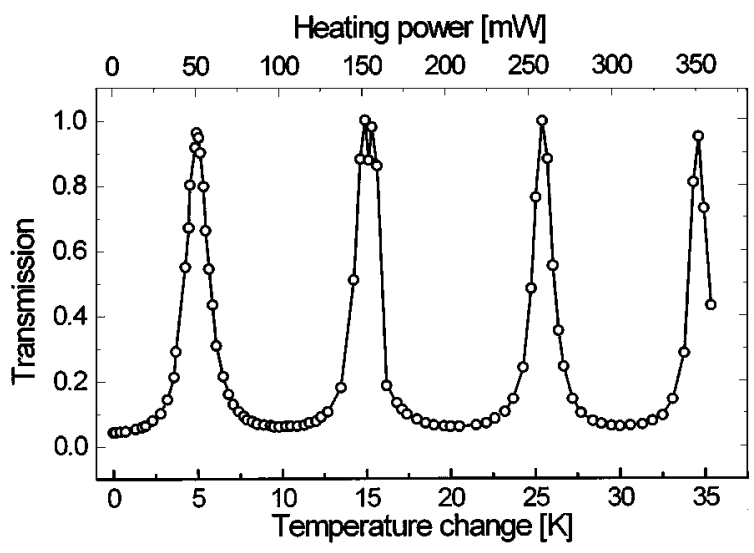

Fig. 2. Temperature dependence of the transmission of the silicon filter.

the couplers, the air gap, and the filter is $\sim 1.5 \mathrm{~dB}$ at the transmission maximum of the filter.

Drifts of the transmitter wavelength can be monitored by measuring the temperature of the filter when its transmission is kept constant. For this purpose, another thin-film resistor made of molybdenum was deposited on the chip. The temperature dependence of the sensor resistor and the shift of the transmission fringe with temperature were determined using a small temperature sensor (LM45) glued on the chip. The transmission peak of the filter was found to shift with temperature by about $11 \mathrm{GHz} / \mathrm{K}$ when current was fed through the heating resistor. The measured transmission as a function of the temperature of the filter chip is shown in Fig. 2. The nominal resistance of the sensing resistor is $\sim 1.7 \mathrm{k} \Omega$ and its measured temperature sensitivity $\sim 3.6 \Omega / \mathrm{K}$. Variations of the ambient temperature in the range of $27^{\circ} \mathrm{C}$ to $40{ }^{\circ} \mathrm{C}$ did not affect the performance of the device. The operating temperature was kept at about 15 $\mathrm{K}$ above the ambient temperature and the required electrical heating power was $150 \mathrm{~mW}$.

\section{MEASUREMENTS}

The system performance of the DFB laser (Lucent 2500 series) with and without the filter was tested in a single channel of a DWDM link. The transmitter was directly modulated in a $2^{31}-1$ bit-long pseudorandom binary sequence at a bit rate of $2.5 \mathrm{~Gb} / \mathrm{s}$. The nominal output power from the DFB laser was

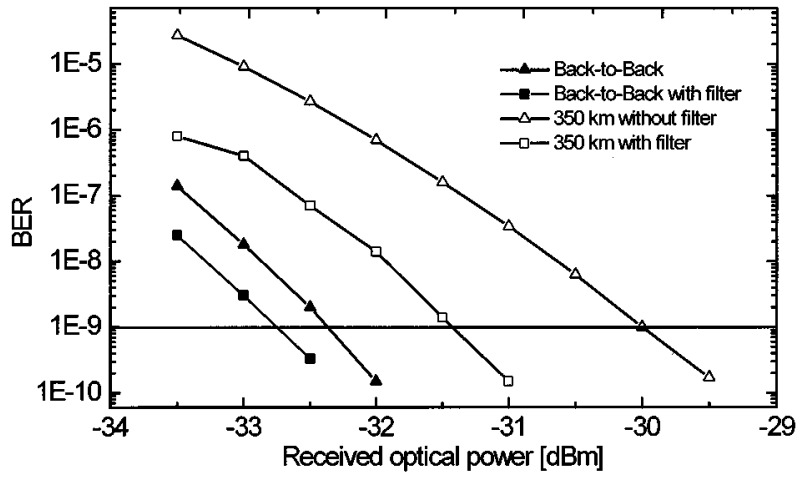

Fig. 3. System performance with and without the filter. The operation point was tuned to $50 \%$ transmission.

$1 \mathrm{dBm}$. The filter was placed directly after the transmitter and the bit error ratio (BER) performance of the system was tested at several operation points along the positive slope of the filter transmission curve. A 350-km-long single-mode fiber link was used in the test. Three optical amplifiers at 100, 175, and 300 $\mathrm{km}$ were applied to keep the ASE noise level low. The output power of the amplifiers was kept at such a level that nonlinear optical effects were negligible. After the link, the signal was detected with a receiver having an avalanche photodetector.

The extinction ratio of the transmitter without the filter was measured by using a digital sampling oscilloscope (HP $54750 \mathrm{~A}$ ) and an optical receiver module (HP83486). Without the filter, the extinction ratio was found to be $9.2 \mathrm{~dB}$ and insertion of the filter improved the ratio to $\sim 13.3 \mathrm{~dB}$. This value depends on the selection of the operation point. We measured the frequency chirp of the laser transmitter with a Fabry-Perot etalon as a frequency discriminator [5]. The adiabatic frequency difference between the ON and OFF states was $\sim 4 \mathrm{GHz}$. Results of the corresponding BER measurement are given in Fig. 3. The measurements were made with and without the filter. For our transmitter, the best performance was obtained when the operation point was tuned to around $50 \%$ of the transmission maximum. The dispersion penalty was improved from $2.4 \mathrm{~dB}$ to $0.9 \mathrm{~dB}$ measured at a BER level of $10^{-9}$.

The waveform of the signal was measured with a digital sampling oscilloscope and a fast photodiode (HP11982). The waveforms after the link with and without the filter are shown in Fig. 4. The signals at the one level are almost identical whereas the power at the zero level has clearly decreased. This result indicates that the reduced dispersion penalty can be partly explained by the improved extinction ratio. It can also be noted that the relaxation oscillations at the beginning of the pulse are higher with filtering than without it, as is expected for an operation point, which is at the positive slope of the transmission spectrum of the filter.

When the wavelength of the transmitter varies, the control system tunes the optical thickness of the filter via the temperature in order to keep the transmission constant. The required temperature change was found to be linearly related to the change in the sensor resistance. A calibration curve for the resistance-versus-wavelength relationship is shown in Fig. 5. A tunable laser source was used to perform the wavelength scan. 


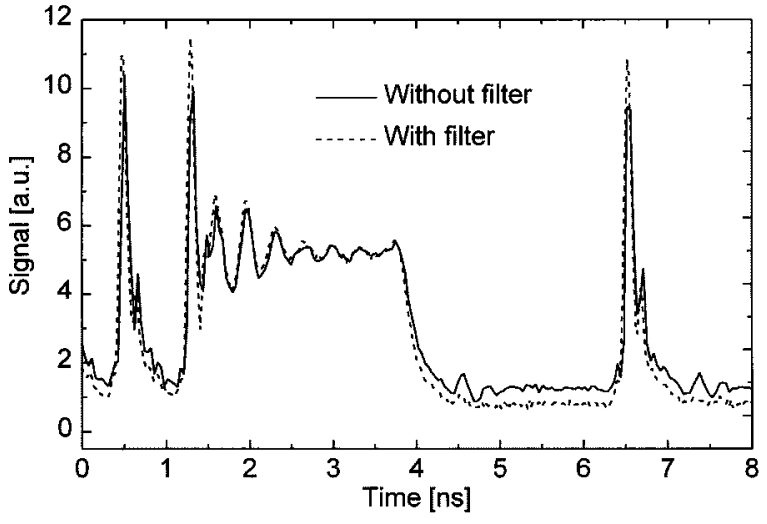

Fig. 4. Waveforms of the signal after the link with and without spectral filtering.

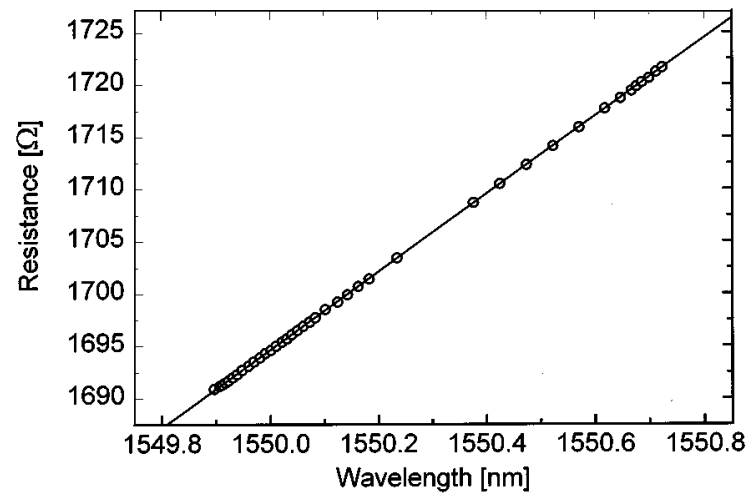

Fig. 5. Relationship between the wavelength and the resistance of the temperature sensor.

The wavelength of the laser was measured with a wavemeter and the resistance with a precision multimeter. The sensitivity of the resistance for the wavelength change is $37 \Omega / \mathrm{nm}$. In a separate test measurement, the filter was found to be capable of tracking the wavelength over a range of $30 \mathrm{~nm}$. An estimate for the accuracy of the wavelength measurement was done by making a linear fit to the measured data. The standard deviation of the fit is less than $2 \mathrm{pm}$, which is $0.25 \%$ of the channel spacing in a $100-\mathrm{GHz}$ DWDM system.

\section{CONCLUSION}

We have introduced a tunable silicon etalon for filtering the output of directly modulated DFB lasers used in DWDM transmitter applications. The operation point of the filter is tuned by changing the temperature of the chip. Two integrated thin-film resistors allow simple implementation for controlling and sensing the temperature of the chip. By inserting the filter directly after the output of a DFB laser, the dispersion effects can be reduced and the transmission distance of a DWDM transmitter can be increased. The changes in the operating wavelength of the transmitter can be continuously monitored by detecting the temperature changes of the filter chip when the operation point on the transmission spectrum is locked. The temperature variations can be linearly related to the changes in the wavelength. A test measurement with $350 \mathrm{~km}$ of standard single-mode fiber demonstrated that the spectral filtering improved the dispersion penalty from 2.4 to $0.9 \mathrm{~dB}$. The wavelength of the laser could simultaneously be monitored in real time with an accuracy on the order of a few picometers.

\section{ACKNOWLEDGMENT}

The authors would like to thank Nokia Networks and Nokia Research Center for generous loan of equipment.

\section{REFERENCES}

[1] C. H. Lee, S. S. Lee, H. K. Kim, and J. H. Han, "Transmission of directly modulated $2.5 \mathrm{~Gb} / \mathrm{s}$ signals over $250-\mathrm{km}$ of nondispersion-shifted fiber by using a spectral filtering method," Photon. Technol. Lett., vol. 8, pp. 1725-1727, Dec. 1996.

[2] P. A. Morton, G. E. Shtengel, L. D. Tzeng, R. D. Yadvish, T. Tanbun-Ek, and R. A. Logan, " $38.5 \mathrm{~km}$ error free transmission at $10 \mathrm{Gbit} / \mathrm{s}$ in standard fiber using a low chirp, spectrally filtered, directly modulated 1.55 $\mu \mathrm{m}$ DFB laser," Electron. Lett., vol. 33, pp. 310-311, 1997.

[3] K. Inoue, "Optical filtering to reduce chirping influence in LD wavelength conversion," Photon. Technol. Lett., vol. 8, pp. 770-772, June 1996.

[4] H. Y. Yu, D. Mahgerefteh, P. S. Cho, and J. Goldhar, "Improved transmission of chirped signals from semiconductor optical devices by pulse reshaping using a fiber Bragg grating filter," J. Lightwave Technol., vol. 17, pp. 898-903, May 1999.

[5] T. Niemi, S. Tammela, T. Kajava, M. Kaivola, and H. Ludvigsen, "Temperature-tunable silicon-wafer etalon for frequency chirp measurements," Microw. Opt. Technol. Lett., vol. 20, pp. 190-192, Feb. 1999.

[6] T. Kajava, M. Kaivola, H. Ludvigsen, T. Niemi, and S. Tammela, "Tunable Fabry-Perot micro-filters for telecommunication system diagnostics," presented at the Tech. Dig. Conf. Lasers and Electro-Optics CLEO/Europe'98, Glasgow, Scotland, IEEE Catalog Number 98TH8326, Paper CThH108. 